\title{
Clinical results of microfluidic antibody-independent peripheral blood circulating tumor cell capture for the diagnosis of lung cancer
}

\author{
Eric Lim, MD, FRCS, ${ }^{\text {a,b }}$ Andee Tay, BSc, ${ }^{\mathrm{b}}$ Jan Von Der Thusen, MD, PhD, ${ }^{\mathrm{a}, \mathrm{c}}$ Maxim B. Freidin, PhD, ${ }^{\mathrm{a}, \mathrm{b}}$ \\ Vladimir Anikin, MD, FRCS, ${ }^{\mathrm{b}}$ and Andrew G. Nicholson, DM, FRCPath ${ }^{\mathrm{a}, \mathrm{c}}$
}

\begin{abstract}
Objectives: The ability to capture and characterize peripheral blood circulating tumor cells has the potential for the development of a blood test for cancer. The aim of this study was to evaluate the diagnostic performance of microfluidic technology as a proof-of-concept study.
\end{abstract}

\begin{abstract}
Methods: Blood from patients undergoing surgery for known or suspected lung cancer was obtained and processed using a microfluidic biochip. Diagnostic performance was evaluated against the reference of cancer identified within surgically obtained formalin-fixed paraffin embedded specimens reported by a principal pathologist. Agreement was assessed in a sample reported by a second independent pathologist. Sensitivity- and specificity-weighted analyses were undertaken.
\end{abstract}

\begin{abstract}
Results: From March 2011 to October 2012, 46 patients at our institution donated blood for research. Cancer was the underlying diagnosis in $43(94 \%) ; 34(79 \%)$ of the patients had primary lung cancer. The proportion of patients with cancer in which atypical cells suspicious for cancer were identified on hematoxylin and eosin staining was $16 / 43(37 \%)$ by the principal pathologist and $10 / 17(59 \%)$ by the second pathologist. On sensitivity-weighted analysis, the sensitivity of the biochip was 54\% (95\% confidence interval [CI], 37-72) and the specificity was $33 \%(95 \% \mathrm{CI}, 2-91)$. On specificity-weighted analysis, the sensitivity was $43 \%$ (95\% CI, 21-71) and the specificity was 100\% (95\% CI, 5-100).
\end{abstract}

Conclusions: This work highlights the potential of microfluidic technology to develop a blood test for the diagnosis of cancer using peripheral blood; conventional clinical criteria can be used as a proof-of-concept of what may be possible with today's technology. (J Thorac Cardiovasc Surg 2014;147:1936-8)

The ability to capture and characterize circulating tumor cells (CTCs) has the potential to lead to clinical applications in cancer that include disease monitoring, refining prognosis, and predicting treatment response.

Several different technologies are being developed to capture peripheral blood CTCs either by size filtration, antibody recognition (followed by immunomagnetic separation), or direct blood smear analysis. ${ }^{1}$ Indirect identification of CTCs in peripheral blood by real-time polymerase chain reaction is also possible. ${ }^{2}$ Recent advances in

From the National Heart and Lung Division, ${ }^{\mathrm{a}}$ Imperial College, London; Academic Division of Thoracic Surgery ${ }^{\mathrm{b}}$ and Department of Histopathology, ${ }^{\mathrm{c}}$ The Royal Brompton and Harefield Hospitals NHS Foundation Trust, London, United Kingdom.

Andee Tay was supported by a PhD fellowship from Point Hope Group Singapore. The project was funded by Professor Peter Goldstraw and supported by the National Institute of Health Research Respiratory Disease Biomedical Research Unit at the Royal Brompton and Harefield NHS Foundation Trust and Imperial College London.

Disclosures: Eric Lim reports consulting fees from Abbott Molecular, Strategen, and GlaxoSmithKline, and lecture fees from Roche and Imedex. All other authors have nothing to disclose with regard to commercial support.

Received for publication June 6, 2013; revisions received Aug 31, 2013; accepted for publication Sept 25, 2013; available ahead of print Dec 2, 2013.

Address for reprints: Eric Lim, MD, FRCS, The Academic Division of Thoracic Surgery, Royal Brompton Hospital, Sydney St, London SW3 6NP, United Kingdom (E-mail: e.lim@rbht.nhs.uk).

$0022-5223 / \$ 36.00$

Copyright (C) 2014 by The American Association for Thoracic Surgery

http://dx.doi.org/10.1016/j.jtcvs.2013.09.052 microfluidic engineering have facilitated the development of an antibody-independent, microfluidic, biochip designed to capture peripheral blood CTCs. ${ }^{3}$ Initial tests developed using spiked in cancer cells (from cell lines) were undertaken with good results, however the technology has yet to be evaluated in a clinical setting.

In this study, the microfluidic biochip was evaluated to determine if it is possible to capture and identify cancer cells circulating in the blood of patients using conventional cytomorphologic criteria with hematoxylin and eosin (H\&E) staining as a proof-of-concept study in a series of patients undergoing surgery for lung cancer.

\section{METHODS}

The study was undertaken at The Royal Brompton and Harefield NHS Foundation Trust. The project was approved under the auspices of the National Institute of Health Biomedical Research Unit Advanced Disease Biobank (NRES 10/H0504/9).

From March 2011 to February 2012, blood samples were obtained from 46 patients and processed using the ClearCell CTChip (Clearbridge BioMedics, Singapore) by filtering $1 \mathrm{~mL}$ of blood within a microfluidic biochip and trapping cells within the 900 chambers; the samples were subsequently stained using $\mathrm{H} \& \mathrm{E}$ and reported in-chip ${ }^{3}$ as previously described. ${ }^{4}$ Patient blood samples were acquired before surgery (by venesection or central venous catheter) and samples were processed on the day of receipt.

After H\&E staining, the biochips were assessed by a principal pathologist (A.G.N). When present, enlarged nucleated cells, either single or in clusters, were classified as atypical cells suspicious for cancer. 


\section{Abbreviations and Acronyms \\ $\mathrm{CTC}=$ circulating tumor cell \\ $\mathrm{H} \& \mathrm{E}=$ hematoxylin and eosin \\ $\mathrm{SD}=$ standard deviation}

To assess interobserver agreement, 18 biochips were also reviewed in a blinded fashion independently by a second pathologist. Agreement was reported by means of a kappa statistic and degree of agreement was classified according to the criteria of Landis and $\mathrm{Koch}^{5}$ : less than 0, no agreement; 0.0 to 0.20 , slight agreement, 0.21 to 0.40 , fair agreement; 0.41 to 0.60 , moderate agreement; 0.61 to 0.80 , substantial agreement; 0.81 to 1.00 , almost perfect agreement.

The diagnostic performance of the microfluidic system was evaluated by sensitivity, specificity, and positive and negative predictive values against the reference standard of cancer identified within surgically obtained formalin-fixed paraffin embedded biopsy or resection specimens of lung. Exploratory analyses were undertaken to help determine the optimum clinical classification: a sensitivity-weighted analysis (either pathologist classifies the results as suspicious or positive for cancer) and a specificity-weighted analysis (both pathologists classify the results as suspicious or positive for cancer).

Categorical data are presented as frequencies (\%) and continuous data are presented as means with standard deviation (SD). Statistical analyses were performed using Stata version 10.0 (StataCorp, College Station, Tex).

\section{RESULTS}

From March 2011 to October 2012, 46 patients at our institution donated blood for research (Table 1). The mean age was 66 years (SD 12 years) and $22(48 \%)$ were men. Cancer was present as an underlying diagnosis in 43 (94\%); 34 (79\%) had primary lung cancer and metastatic cancer of nonlung origin was diagnosed in $8(19 \%)$. Of the 43 patients with cancer, $18(42 \%)$ had stage I to II tumors and $22(51 \%)$ had stage III to IV tumors. In 3 cases, the stage of the tumor was not identified. The size of $60 \%$ of tumors was category $\mathrm{T} 1$ or $\mathrm{T} 2 ; 46 \%$ were lymph node positive and $49 \%$ were lymph node negative.

The proportion of patients with cancer in which atypical cells suspicious for cancer were identified on $\mathrm{H} \& \mathrm{E}$ staining was $16 / 43(37 \%)$ by the principal pathologist and 10/17 $(59 \%)$ by the second pathologist. Each pathologist also identified the cells in 1 of the 3 patients with benign disease they tested. The interobserver agreement was $80 \%$ corresponding to a kappa of 0.615 indicating substantial agreement.

Staging was reported clinically in 40 of 43 patients with cancer as a diagnosis (Table 1). Among these 40 patients, atypical cells suspicious for cancer on conventional light microscopy were identified in $6 / 18(33 \%)$ patients with early stage (I/II) and 10/22 (45\%) patients with advanced stage (III/IV) disease.

Using a sensitivity-weighted analysis, the test performance for the diagnosis of cancer yielded a sensitivity of $54 \%$ (95\% confidence interval [CI], 37-72), specificity of $33 \%$ (95\% CI, 2-91), a negative predictive value of $4 \%$ (95\% CI, 1-19), and a positive predictive value of $93 \%$
(95\% CI, 89-97). Using a specificity-weighted analysis, the test performance for the diagnosis of cancer yielded a sensitivity of $43 \%$ (95\% CI, 21-71), specificity of $100 \%$ (95\% CI, 5-100), a negative predictive value of $7 \%$ (95\% CI, 2-29), and a positive predictive value of $95 \%$ (95\% CI, 85-98).

\section{DISCUSSION}

Microfluidics is considered to be one of the most promising technologies to capture CTCs. ${ }^{6}$ The advantage of microfluidic methods is that they can simultaneously take into account the physical and biological properties of CTCs, such as size and expression of tumor-specific biomarkers. Different microfluidic approaches are being developed and tested experimentally. This study assessed the size-filtration technique using a ClearCell CTChip (Figure 1), which consists of a chamber with 900 crescent-shaped pillar traps that can catch enlarged and less-deformable (stiffer) tumor cells, while allowing red blood cells and leukocytes to go through. ${ }^{3}$ The biological features of the entrapped cells can be analyzed subsequently using appropriate staining (including immunocytochemistry); the cells can also be retrieved from the chip and used for downstream applications such as gene expression analysis, mutation detection, and cell culture.

TABLE 1. Baseline characteristics of the patients

\begin{tabular}{|c|c|}
\hline Characteristic & Value \\
\hline Sample size, $\mathrm{n}$ & 46 \\
\hline Mean age, y (SD) & $66(12)$ \\
\hline Men, n (\%) & $22(48)$ \\
\hline Cancer as an underlying diagnosis, $\mathrm{n}(\%)$ & $43(94)$ \\
\hline Primary lung cancer, n (\%) & $34(79)$ \\
\hline Metastatic lung cancer, n (\%) & $8(19)$ \\
\hline Other primary thoracic malignancy, $\mathrm{n}(\%)$ & $1(2)$ \\
\hline Benign lung disease, $\mathrm{n}(\%)$ & $3(6)$ \\
\hline \multicolumn{2}{|l|}{ Tumor grade } \\
\hline Stage I to II, n (\%) & $18(42)$ \\
\hline Stage III to IV, n (\%) & $22(51)$ \\
\hline Insufficient information to classify stage, $\mathrm{n}(\%)$ & $3(7)$ \\
\hline \multicolumn{2}{|l|}{ Tumor size } \\
\hline $\mathrm{T} 1, \mathrm{n}(\%)$ & $13(30)$ \\
\hline $\mathrm{T} 2, \mathrm{n}(\%)$ & $13(30)$ \\
\hline $\mathrm{T} 3, \mathrm{n}(\%)$ & $2(5)$ \\
\hline $\mathrm{T} 4, \mathrm{n}(\%)$ & $5(12)$ \\
\hline Unknown, n (\%) & $10(23)$ \\
\hline \multicolumn{2}{|l|}{ Lymph node status } \\
\hline Positive, n (\%) & $20(46)$ \\
\hline Negative, n (\%) & $21(49)$ \\
\hline Unknown, n (\%) & $2(5)$ \\
\hline \multicolumn{2}{|l|}{ Smoking history } \\
\hline Current smoker, n (\%) & $10(22)$ \\
\hline Ex-smoker, n (\%) & $16(35)$ \\
\hline Never smoker, n (\%) & $6(13)$ \\
\hline Unknown, n (\%) & $14(30)$ \\
\hline
\end{tabular}




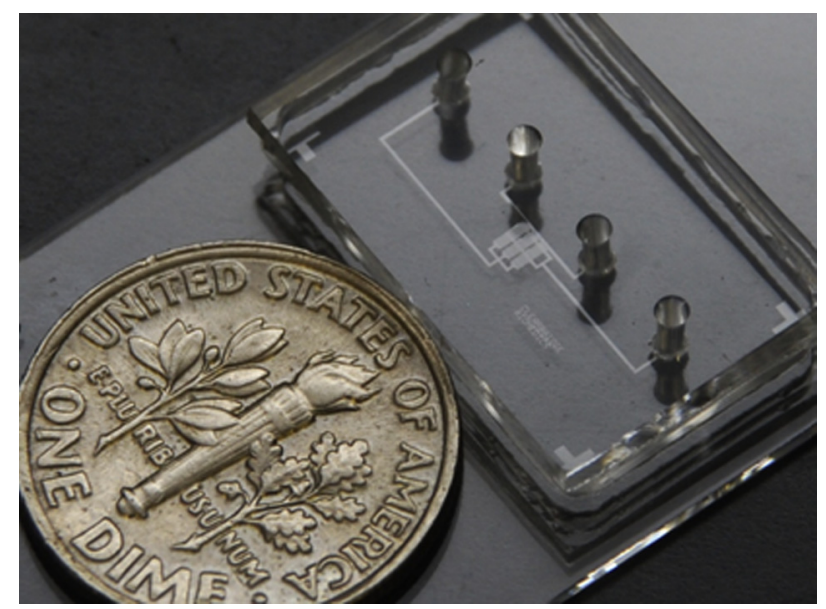

FIGURE 1. Microfluidic biopchip next to a US 10 cent coin.

Our study is the first clinical report in which microfluidic technology was used to capture atypical cells from peripheral blood by staining them using routine histologic stains and examining them using light microscopy. Although the clinical test performance was not perfect for sensitivity, excellent specificity was achieved using $1 \mathrm{~mL}$ of blood for diagnosing lung cancer. The samples analyzed were collected in a nonbiased prospective way without preliminary selection of patients by any criteria. Therefore, we believe that the sample reflects a common situation for clinicians in daily practice. As a drawback, the sample contains only 3 patients with benign lung disease, hence the wide confidence limits with the estimates of specificity.

Currently, a panel of antibody stains (such as EpCAM+, CD45-, DAPI+) are commonly applied to diagnose lung cancer using CTCs. However, this is not the accepted clinical definition as published by the World Health Organization $^{7}$ and epithelial (nonmalignant) cells also carry the same immunophenotype. ${ }^{8}$ In an effort to develop a point-of-care blood-based diagnostic test, our focus is on replicating conditions that would facilitate conventional clinical diagnosis from blood; microfluidic technology seems to provide a (small) advance on current technology that cannot differentiate epithelial and cancer cell capture.

With regard to potential clinical application, the current test performance (relatively low sensitivity) suggests that as a negative result cannot rule out disease, the high specificity implies that patients who test positive most likely do have the disease. The test sensitivity is influenced by the number of patients (with suspected lung cancer) with CTCs in the blood, a parameter this is not well studied (and not under our control), and may be affected by the small amount of blood $(1 \mathrm{~mL})$ used. In addition, analysis and reporting of cells within a microfluidic chamber is a completely new method of reporting on cells that have been subject to shear stresses. Based on our data, we estimate that $33 \%$ of patients with early cancer and $45 \%$ of patients with advanced cancer have atypical cells identifiable in their peripheral blood. If this is the case, successful clinical implementation could define this as the proportion of patients who would not need to undergo confirmatory tissue biopsy. Currently, our estimates have a wide confidence interval because it was a proof-ofconcept study.

At present, this technology is limited by an inability to definitively confirm that the atypical cells are from the primary tumor, but given their morphology, it seems likely that this is the case as the cells are larger than circulating lymphocytes and other white blood cells. The shear stress rendered from the microfluidic cell capture distorts the architecture of the cells and having to undertake light microscopy though a biochip is an additional a limitation. This may be one of the main reasons for disagreement between the pathologists in identification of the CTCs ( $20 \%$ in our study). Further refinements are underway to ensure better cell preservation and complete cell extraction with the application of immunocytochemistry (where required) to further characterize the captured cells in our efforts to achieve a clinical grade diagnostic test.

\section{CONCLUSIONS}

Our results demonstrate the potential of microfluidic technology to develop a highly specific blood test for the diagnosis of cancer in peripheral blood; conventional clinical criteria can be used as proof-of-concept of what can be achieved with today's technology.

\section{References}

1. Pantel K, Brakenhoff RH, Brandt B. Detection, clinical relevance and specific biological properties of disseminating tumour cells. Nat Rev Cancer. 2008;8: 329-40.

2. Yamashita J, Matsuo A, Kurusu Y, Saishoji T, Hayashi N, Ogawa M. Preoperative evidence of circulating tumor cells by means of reverse transcriptase-polymerase chain reaction for carcinoembryonic antigen messenger RNA is an independent predictor of survival in non-small cell lung cancer: a prospective study. $J$ Thorac Cardiovasc Surg. 2002;124:299-305.

3. Tan SJ, Yobas L, Lee GY, Ong CN, Lim CT. Microdevice for the isolation and enumeration of cancer cells from blood. Biomed Microdevices. 2009;11:883-92.

4. Lim E, Tay A, Nicholson AG. Antibody independent microfluidic cell capture of circulating tumor cells for the diagnosis of cancer. J Thorac Oncol. 2012;7:e42-3.

5. Landis JR, Koch GG. The measurement of observer agreement for categorical data. Biometrics. 1977;33:159-74.

6. Dong Y, Skelley AM, Merdek KD, Sprott KM, Jiang C, Pierceall WE, et al. Microfluidics and circulating tumor cells. J Mol Diagn. 2013;15:149-57.

7. Travis WD, Müller-Hermelink HK, Harris CC, Brambilla E. Pathology and Genetics of Tumours of the Lung, Pleura, Thymus and Heart. Geneva: IARC Press; 2004.

8. Tanaka F, Yoneda K, Kondo N, Hashimoto M, Takuwa T, Matsumoto S, et al. Circulating tumor cell as a diagnostic marker in primary lung cancer. Clin Cancer Res. 2009;15:6980-6. 